\title{
Improving the Classification of Traumatic Brain Injury: The Mayo Classification System for Traumatic Brain Injury Severity
}

\section{Daniel Philip Friedland*}

MA Clinical Psychology, Consultant Clinical Psychologist/Neuropsychologist, Acquired Brain Injury Team, UK

\begin{abstract}
This paper discusses the Mayo Classification System for Traumatic Brain Injury Severity. This system is viewed as an innovative system which distinguishes between different severities of traumatic brain injury in keeping with the general outcome literature regarding traumatic brain injury. In addition, this system emphasises the probability that the brain has been injured. This is a unique feature in traumatic brain injury classification systems, and has practical implications for rehabilitation.
\end{abstract}

Keywords: Classification; Traumatic; Brain; Injury; Mayo; Severity

\section{Introduction}

Traumatic brain injury (TBI) has been defined as "an alteration in brain function, or other evidence of brain pathology, caused by an external force" [1]. Alteration in brain function is defined as one of the following clinical signs: any period of loss of consciousness, any loss of memory for events immediately before (retrograde amnesia) or after the injury (post-traumatic amnesia/PTA), neurological deficits, and alteration in mental state at the time of injury e.g. confusion/ disorientation [1].

\section{Classification of TBI}

Classification of TBI is based on the length of loss of consciousness, Glasgow Coma Scale (GCS) score and length of post-traumatic amnesia. A GCS score of 13-15 is characterised as a mild TBI, a GCS of 9-12 is categorised as a moderate TBI, and a GCS of 3-8 is characterised as a severe TBI [2].

Post-traumatic amnesia is an important predictor of functional outcome [3]. Levin defined post-traumatic amnesia as the interval during which the patient is confused, amnesic for ongoing events and likely to show behavioural disturbance [4]. More recently posttraumatic amnesia has been defined as the interval from the injury until the patient is orientated and can form and later recall new memories [5]. A PTA up to 24 hours has been categorised as a mild TBI, a PTA between 1-7 days a moderate TBI, and PTA beyond 7 days has been characterised as a severe TBI [6].

Further indicators of TBI severity include the presence of abnormalities shown on neuroimaging including haematoma, contusion, diffuse axonal injury, and haemorrhages [7]. The classification of the severity of a traumatic brain injury (TBI) is important as it helps to guide acute medical management [8]. Classification of TBI also helps guide subsequent neuro rehabilitation, and may help to determine whether in-patient or community neuro rehabilitation is required $[9,10]$. A consistent and reliable TBI classification system is also important in TBI research [7].

\section{Challenges in Classifying TBI according to Single Indicators}

It is important to note that none of the TBI indicators is a 'gold standard' for determining TBI severity, and there are limitations with regards to each of these indicators. For example, there are limitations with neuroimaging. Neuroimaging can underestimate the extent of diffuse axonal injury [10]. The timing of neuroimaging can have an impact on whether trauma-related abnormalities may be detected. An individual may have an abnormal brain scan at 7 days post- injury but not at 12 months post- injury [11]. Thus, if the individual was only scanned at 12 months post-injury the neuroimaging may suggest that a TBI did not occur when this is clearly not the case.

In terms of PTA, an individual may have a very brief period of posttraumatic amnesia but may have abnormal neuroimaging indicative of a TBI. Thus, if one relied on length of PTA the severity of the TBI could be underestimated. The use of analgesics, and more specifically opiate analgesics, can affect cognition and memory in the absence of a traumatic brain injury. This is even the case in the prospective assessment of post-traumatic amnesia. McCarter et al. tested 17 non-head injured, non-traumatic, orthopaedic patients in hospital on Westmead PTA Scale. Only $20 \%$ of participants tested over 4 days reached the criteria of three 12/12 scores [12]. Therefore individuals who have not sustained a traumatic brain injury and who have been given opiate analgesics can present as being in post-traumatic amnesia in the hospital setting leading to possible incorrect diagnosis of a traumatic brain injury.

The retrospective analysis of post-traumatic amnesia can also yield false positives in terms of diagnosing an individual as suffering from a TBI based on retrospective analysis of post-traumatic amnesia. Kemp et al. [13] study involved 63 orthopaedic patients who had not suffered a traumatic brain injury. They were followed up at about 10 weeks post injury. The Rivermead PTA protocol was used. 38\% reported posttraumatic amnesia like phenomenon despite not having suffered a traumatic brain injury. $30 \%$ of these individuals reported amnesia of 24 hours or more which would place them in the category of moderate traumatic brain injury despite not suffering a traumatic brain injury [13].

In terms of GCS, interventions used in early medical management of moderate to severe TBI can complicate GCS assessment. Intubation, sedation or paralysing agents, as well as facial injuries can limit the accuracy of GCS ratings.

*Corresponding author: Daniel Philip Friedland, MA Clinical Psychology Consultant Clinical Psychologist/Neuropsychologist, Acquired Brain Injury Team, Jacketts Field, Abbots Langley, Hertfordshire, WD5 OPA, UK, Tel: 01923299124; Fax: 01923299108; E-mail: daniel.friedland@hchs.nhs.uk

Received September 03, 2013; Accepted October 28, 2013; Published October 31, 2013

Citation: Friedland DP (2013) Improving the Classification of Traumatic Brain Injury: The Mayo Classification System for Traumatic Brain Injury Severity. J Spine S4: 005. doi:10.4172/2165-7939.S4-005

Copyright: ( 2013 Friedland DP. This is an open-access article distributed under the terms of the Creative Commons Attribution License, which permits unrestricted use, distribution, and reproduction in any medium, provided the original author and source are credited. 
Thus, relying on one indicator, whether it is the GCS score, length of PTA or neuroimaging, can be problematic and it would therefore make sense to take all of these indicators into account when determining severity of TBI.

\section{The Mayo System}

The Mayo Classification for TBI Severity (Mayo System) includes all of the above indicators i.e. loss of consciousness, Glasgow Coma Scale score, PTA, and presence of abnormalities on neuroimaging. In addition the system also includes the presence of skull fractures. The authors note that this system was developed because single indicators can be unreliable at times e.g. early sedation following TBI can affect GCS in that the reduced GCS score is not due to a TBI but rather the effects of sedation. Systemic or psychological shock and organ failure associated with polytrauma can extend PTA. Furthermore, there can be missing documentation in the medical records regarding an individual's duration of loss of consciousness, Glasgow Coma Scale score or duration of post-traumatic amnesia. By including as much positive evidence as possible this system aims to increase the reliability of this classification system [14].

According to the Mayo System there are three main classifications: Definite Moderate-Severe TBI, Probable Mild TBI (MTBI), and Possible TBI. A classification of a Definite Moderate-Severe TBI would be made if one of the following was present: death due to this TBI, loss of consciousness of 30 minutes or more, post-traumatic amnesia of 24 hours or more, worst Glasgow Coma Scale score in the first 24 hours $<13$ (unless invalidated by factors such as intoxication, sedation, systemic shock). In addition if there was evidence of haematoma, contusion, penetrating TBI, haemorrhage, brain stem injury the TBI would be classified as Definite Moderate-Severe TBI [14].

A classification of Probable MTBI is made if one or more of the following criteria apply: loss of consciousness is momentary to 30 minutes and PTA does not extend beyond 24 hours. If the individual sustains a depressed, basilar, or linear skull fracture (dura intact), then the TBI is still a probable MTBI [14].

A classification of Possible TBI is made if one or more of the following symptoms are present: blurred vision, confusion, dazed, dizziness, focal neurological symptoms, headache or nausea [14].

One of the unique features of the Mayo System in comparison to other TBI classification systems is that this system provides a statement about the probability that the brain has been injured. This may be helpful in care planning. The 'definite' cases will likely need some degree of medical follow-up after the acute period. The 'probable' cases may also require some follow-up but are expected to fully recover. The 'possible' cases are often diagnostically complex so follow-up is likely to focus, at last initially, on determining what kind of care or intervention will be of most benefit to them.

In the Mayo sample, the Mayo System allowed for a much larger classification of cases in Definite Moderate-Severe TBI compared to a single indicator. GCS accounted for $8 \%$ of classifications, loss of consciousness accounted for $15 \%$ of classifications, post-traumatic amnesia accounted for $10 \%$ of classifications, and abnormalities on neuroimaging accounted for $60 \%$ of classifications. $100 \%$ of the sample $(\mathrm{n}=139)$ was classified according to the Mayo criteria i.e. using a combination of all of these indicators.

The sensitivity and specificity for Moderate-Severe TBI was calculated to be $98 \%$ and $96 \%$ respectively (this was an approximate value as there is no gold standard for TBI severity). Of the Probable MTBI there was a greater chance of a false negative as CT scan was only done in $10 \%$ of the cases i.e. there may have been intracranial abnormalities if a CT scan had been performed resulting in a classification of Definite Moderate-Severe TBI. There was also the possibility that there were false negatives in the Possible TBI as CT scans were not done in all cases [7]. Sensitivity and specificity were not provided for MTBI cases in this study.

The Mayo System is consistent with other classification systems including the American Congress of Rehabilitation Medicine, Centre for Disease Control, and World Health Organisation which classify MTBI as one in which the individual is not unconscious for greater than 30 minutes, and post-traumatic amnesia does not extend beyond 24 hours [15-17]. This would contribute to validity of the Mayo System as a classification system for TBI severity.

The Mayo System's classification of Possible TBI is an important category. There is an increasing body of literature to show that symptoms such as headaches and cognitive difficulties are not specific to TBI but occur in a range of conditions including chronic pain, depression, and anxiety disorders [18-20]. Recently Donnell et al. [4] looked at the incidence of postconcussion symptoms in psychiatric groups, individuals who had sustained a mild traumatic brain injury and comorbid conditions. In a sample of 4462 randomly sampled US Army veterans who served in Vietnam, only $32 \%$ of veterans with a history of MTBI met the DSM-IV criteria of post-concussion syndrome. A higher incidence of those who did not suffer a TBI but were diagnosed with Post-Traumatic Stress Disorder (40\%), Generalised Anxiety Disorder (50\%) and Major Depressive Disorder (57\%) met the criteria for post-concussion syndrome in the DSM-IV [18]. In other words postconcussion symptoms are not specific to traumatic brain injury. Thus, the Mayo System classification of Possible TBI is consistent with the literature regarding the non-specificity of symptoms such as headaches and reported cognitive difficulties.

\section{The Mayo System in Current Research and Clinical Practice}

The Mayo System has been used in TBI research. For example the sample group in a study of white matter changes following MTBI was based on the Mayo System [21]. The Mayo System has been used in epidemiological studies of TBI [22]. The Mayo System has also been used in research looking at neuropsychological assessment issues $[23,24]$. The Mayo system is used as part post acute clinical evaluation of patients with TBI [14]. The current author has used the Mayo system in relation to research into falls and TBI, and return to work rates following moderate-severe TBI $[25,26]$.

\section{Limitations of the Mayo System}

There are some limitations of the Mayo System. For example, a Definite Moderate-Severe TBI would be diagnosed if post-traumatic amnesia extended beyond 24 hours. However, as noted earlier in this paper the retrospective analysis of post-traumatic amnesia can be complicated by factors such as the use of analgesics following the injury. Post-traumatic amnesia can be overestimated in such cases [17]. Thus, it may be difficult to be certain whether post-traumatic did extend beyond 24 hours, and hence whether a TBI was Probably Mild or Definitely Moderate-Severe within the Mayo System i.e. The Mayo system is not able to get round some of the difficulties inherent in TBI classification especially at the milder end of the spectrum.

Although there are a very limited number of TBI classification systems which incorporate multiple indicators, The Mayo System has 
Citation: Friedland DP (2013) Improving the Classification of Traumatic Brain Injury: The Mayo Classification System for Traumatic Brain Injury Severity. J Spine S4: 005. doi:10.4172/2165-7939.S4-005

also not been compared to other classification systems such as the system in the Department of Veterans Affairs and The Department of Defense system [27].

A limitation of the Mayo System is that it does not differentiate between moderate and severe TBI. This is an important distinction in so far as the outcome following moderate or severe TBI can vary significantly $[5,28]$. Thus, for a more fine grained approach to outcome following moderate-severe TBI other classification systems may need to be employed.

\section{Conclusion}

In conclusion the Mayo System is an innovative classification system which makes use of as much positive evidence as possible and emphasises the probability of the brain having been injured. This system may be seen as advancement on classification systems which only rely on one indicator, and where there is no reference to the likelihood of a brain injury. The Mayo System manages to use multiple indicators and is in keeping with current literature on outcome following TBI. This system may be applied in both research and clinical practise in neurorehabilitation and TBI. Further research into the validity of this system is recommended.

\section{Acknowledgements}

I would like to thank Dr. Malec for his helpful suggestions regarding this paper, and in particular the practical applications of the probabilistic model of TBI.

\section{References}

1. Menon DK, Schwab K, Wright DW, Maas Al (2010) Demographics and Clinical Assessment Working Group of the International and Interagency Initiative toward Common Data Elements for Research on Traumatic Brain Injury and Psychological Health Position statement: definition of traumatic brain injury. Arch Phys Med Rehabil 91: 1637-1640.

2. Teasdale G, Jennett B (1974)Assessment of coma and impaired consciousness A practical scale. Lancet 2: 81-84.

3. Sherer M, Struchen MA, Yablon SA, Wang Y, Nick TG (2008) Comparison of indices of traumatic brain injury severity: Glasgow Coma Scale, length of coma and post-traumatic amnesia. J Neurol Neurosurg Psychiatry 79: 678-685.

4. Levin HS, O'Donnell VM, Grossman RG (1979) The Galveston Orientation and Amnesia Test. A practical scale to assess cognition after head injury. J Nerv Ment Dis 167: 675-684.

5. Nakase Richardson R, Sherer M, Seel RT, Hart T, Hanks R, et al. (2011) Utility of post-traumatic amnesia in predicting 1-year productivity following traumatic brain injury: comparison of the Russell and Mississippi PTA classification intervals. J Neurol Neurosurg Psychiatry 82: 494-499.

6. Stein S (1996) Classification of head injury. In: Narayan R, Povlishock J, Wilberger J (eds). Neurotrauma. McGraw-Hill.

7. Saatman KE, Duhaime AC, Bullock R, Maas Al, Valadka A, et al. (2008) Classification of traumatic brain injury for targeted therapies. J Neurotrauma 25: 719-738.

8. (2003) National Institute for Clinical Excellence. Head Injury: triage, assessment, investigation and early management of head injury in infants, children and adults. London: National Institute for Clinical Excellence.

9. Friedland D, Hutchinson P (2013) Classification of traumatic brain injury Advances in Clinical Neuroscience \& Rehabilitation 12-13.

10. Azouvi P (2000) Neuroimaging correlates of cognitive and functional outcome after traumatic brain injury. Current Opinion in Neurology 13: 665-669.
11. Moen KG, Skandsen T, Folvik M, Brezova V, Kvistad KA et al. (2012) A longitudinal MRI study of traumatic axonal injury in patients with moderate and severe traumatic brain injury. J Neurology Neurosurgery Psychiatry 12: 11932000.

12. McCarter RJ, Walton NH, Moore C, Ward A, Nelson I (2007) PTA testing, the Westmead post traumatic amnesia scale and opiate analgesia: A cautionary note. Brain Injury 21: 1393-1397.

13. Kemp S, Agostinis, House A, Coughlan A (2010) Analgesia and other causes of amnesia that mimic post-traumatic amnesia (PTA): A cohort study. Journal of Neuropsychology 4: 231-236.

14. Malec JF, Brown AW, Leibson CL, Flaada JT, Mandrekar JN, et al. (2007) The Mayo Classification System for Traumatic Brain Injury Severity. Journal of Neurotrauma 9: 1417-1424.

15. (1993) Mild Traumatic Brain Injury Committee, A.C.o.R.M, Head Injury Interdisciplinary Special Interest Group. Definition of mild traumatic brain injury. Journal of Head Trauma Rehabilitation 3: 86-87.

16. (2003) Report to Congress on Mild Traumatic Brain Injury in the United States: Steps to Prevent a Serious Public Health Problem. Atlanta, GA: National Centre for Injury Prevention and Control, Centre for Diseases Control and Injury Prevention.

17. (1992) World Health Organization. International statistical classification of diseases and related health problems (10thedn) Geneva, Switzerland: World Health Organization.

18. Donnell A, Kim M, Silva M, Vanderploeg R (2012) Incidence of Postconcussion Symptoms in Psychiatric Diagnostic Groups, Mild Traumatic Brain Injury, and Comorbid Conditions. Clinic Neuropsychol 7: 1092-1101.

19. Iverson G, Lange R (2003) Examination of "Postconcussion-Like" Symptoms in a Healthy Sample Applied Neuropsychology 3: 137-144.

20. Smith-Seemiller L, Fow N, Kant R, Franzen M (2003) Presence of postconcussion syndrome symptoms in patients with chronic pain vs mild traumatic brain injury. Brain Injury 17: 199-206.

21. Kinnunen K, Greenwood R, Powell J, Leech R, Hawkins P, et al. (2011) White matter damage and cognitive impairment after traumatic brain injury Brain 134 449-463.

22. Leibson C, Brown C, Ransom J, Diehl N, Perkins P, et al. (2011) Incidence of Traumatic Brain Injury Across the Full Disease Spectrum Epidemiology 22: 836-844.

23. Walker A, Batchelor J, Arthus Shores E, Jones M (2009) Diagnostic efficiency of demographically corrected Wechsler Adult Intelligence Scale-III and Wechsler Memory Scale-III indices in moderate to severe traumatic brain injury and lower education levels. Journal of the International Neuropsychological Society 15: 938-950.

24. Whitney K, Davis J, Shepard P, Bertram D, Adams K (2009) Digit Span Age Scaled Score in Middle-Aged Military Veterans: Is It More Closely Associated with TOMM Failure than Reliable Digit Span? Arch Clin Neuropsychol 24: 263 272.

25. Friedland D, Brunton I, Potts J (2013) Falls and Traumatic Brain Injury in Adults Under the Age of Sixty. Journal of Community Health.

26. Friedland D, Potts J (2013) Return to work following moderate-severe traumatic brain injury: a UK community sample.

27. http://www.dcoe.mil/content/navigation/documents/VA\%20Dod $\% 20$ Management $\% 20$ of\%20Concussion \%20mild\%20Traumatic\%20Brain $\% 20$ Injury\%20Summary.pdf

28. Iverson G, Lange R (2011) Moderate and Severe Traumatic Brain Injury. In Schoenberg M and Scott J (Eds) The Little Black Book of Neuropsychology. New York: Springer. 